ESTABILIDADE TÉRMICA E VAPORIZAÇÃO DO ÓLEO ESSENCIAL DE TOMILHO

\author{
THERMAL STABILITY AND VAPORIZATION OF THYME ESSENTIAL OIL
}

Angela Maria Picolloto ${ }^{1}$; Angela Maria Ariati $^{2}$; Lidaiane Mariah Silva dos Santos Franciscato ${ }^{2}$; João Marcos Azevedo Gomes dos Santos2; Paulo Rodrigo Stival Bittencourt ${ }^{3}$; Elina Basto Caramão ${ }^{4}$; Cristiane Mengue Feniman Moritz ${ }^{2}$

1. Universidade Estadual de Maringá, Departamento de Física

2. Universidade Estadual de Maringá, Departamento de Tecnologia

3. Universidade Tecnológica Federal do Paraná, Departamento de Química

4. Universidade Tiradentes, Instituto de Tecnologia

\title{
RESUMO
}

O presente trabalho teve como objetivo analisar a estabilidade térmica de um óleo essencial comercial de tomilho, submetido a cinco temperaturas diferentes de tratamentos térmicos e dois estados de armazenamento. Utilizou-se as técnicas de Análise Termogravimétrica e Calorimetria Exploratória Diferencial e a estabilidade termoquímica do óleo essencial foi caracterizada por meio de Cromatografia Gasosa e Espectroscopia no Infravermelho por Transformada de Fourier. Houve estabilidade e sem perda de massa do óleo essencial de tomilho até aproximadamente $75{ }^{\circ} \mathrm{C}$. Resultados indicaram que não ocorreu flutuação do comportamento térmico nas amostras estocadas hermeticamente durante os tratamentos térmicos, enquanto que nas amostras acondicionadas em estado aberto, o comportamento térmico foi otimizado até o tratamento a $60{ }^{\circ} \mathrm{C}$. As alterações da composição química e dos grupo funcionais do óleo essencial sob aquecimento e condição de acondicionamento também foram avaliadas.

\section{PALAVRAS CHAVE}

Análise Termogravimétrica; Calorimetria Exploratória Diferencial; Cromatografia Gasosa; Espectrometria de Infravermelho.

\begin{abstract}
Present work aimed to study, evaluate, and compare the thermal stability of a commercial essential oil of thyme, heated to five different heat treatment temperatures and two storage states. The techniques of Thermogravimetric Analysis and Differential Scanning Calorimetry were used and the thermochemical stability of the essential oil was characterized by means of Gas Chromatography and Fourier Transform Infrared Spectroscopy. There was stability and no loss of mass of the thyme essential oil up to approximately $75^{\circ} \mathrm{C}$. Results indicated that there was no fluctuation of the thermal behavior in the samples hermetically stored during the heat treatments, while in the samples stored in an open state, the thermal behavior was optimized until the treatment at $60{ }^{\circ} \mathrm{C}$. Changes in the chemical composition and functional groups of the essential oil under heating and conditioning conditions were also evaluated.
\end{abstract}

\section{KEY WORDS}

Thermogravimetric Analysis; Differential Scanning Calorimetry; Gas Chromatography; Infrared Spectrometry

Revista Gestão e Sustentabilidade Ambiental., v. 11, n. esp, p. 175-189 jan. 2022^




\section{INTRODUÇÃO}

Recentemente está sendo observada uma tendência na alteração no comportamento dos consumidores de alimentos industrializados, sendo que muitos exigem produtos mais seguros e naturais para o consumo em prol de benefícios para saúde. Este fato vem motivando renovação do desenvolvimento de novos produtos com a utilização de matérias primas naturais, e com elevada atividade antioxidante e antimicrobiana em suas formulações. Uma alternativa para satisfazer esta demanda, é a substituição de aditivos sintéticos por óleos essenciais (RIBEIRO-SANTOS et al., 2018).

De acordo com a Organização Internacional de Padronização (ISO - International Organization for Standardization), os óleos essenciais são produtos extraídos a partir de uma matéria-prima natural de origem vegetal, por destilação a vapor, ou por destilação a seco, sucessivo da separação da fase aquosa, sem que haja mudanças da sua composição (SHARMA et al., 2020).

Os óleos essenciais são constituídos por uma mistura complexa de compostos voláteis e solúveis em água, como terpenos, terpenóides e fenilpropanóides. No presente trabalho, estudamos a estabilidade térmica do óleo essencial de tomilho (Thymus vulgaris) gentilmente cedido pela empresa Laszllo. A composição do óleo essencial de tomilho abrange principalmente fenóis como, timol e carvacrol. Estudos recentes (DEL RÉ; JORGE, 2011) demostram que o óleo essencial de tomilho tem poderosa atividade antioxidante e antimicrobiana o qual é muito empregado na produção alimentos, como, hambúrgueres de salmão e alimentos que embalam algas marinhas na fabricação, salsichas, pães (HUANG et al., 2021; DOLEA et al., 2018). Além do timol, o óleo essencial de tomilho tem sua composição constituída também por hidrocarbonetos, álcoois, ésteres, aldeídos e fenóis, que são eles terpenos, taninos, flavonoides e saponinas (SIQUEIRA, 2015).

Dessa forma, se faz importante o estudo da estabilidade térmica do OE de tomilho, já que, muitas vezes os mesmos podem passar processos que envolvam aplicação de calor, podendo alterar características determinantes na qualidade e também na aplicabilidade do óleo. Sendo assim, é relevante estudar as alterações físico-químicas que podem acontecer como o aquecimento do OE de tomilho, assim como bem conhecer o intervalo de temperatura em que ocorre a decomposição termogravimétrica, desidratação e vaporização. Logo, quando se conhece as faixas de temperatura em que ocorre alterações nas propriedades físico-químicas é possível controlar a aplicação do calor nos óleos, os métodos de estocagem e cuidados no transporte (SHEN, KAMDEM; 2015). Na prática,

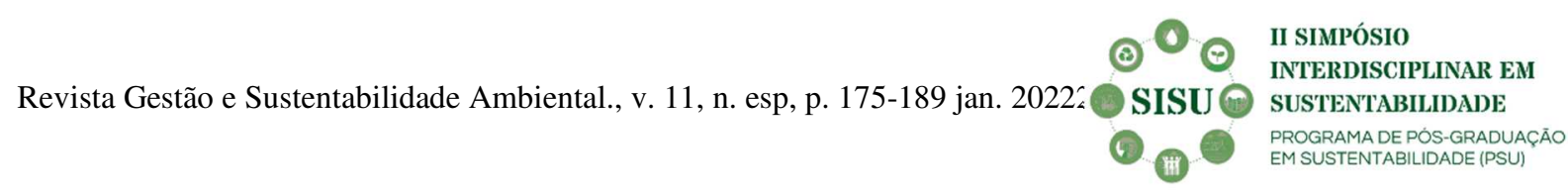


o tratamento térmico nos processos é empregado para viabilizar a eliminação dos micro-organismos contaminantes, retardando os processos microbiológicos e metabólicos, e intensificando a inativação de enzimas. Logo, é importante conhecer o limite de estabilidade térmica do OE, tratado em temperaturas variadas.

Os principais objetivos do presente trabalho foram, primeiramente, analisar e comparar o comportamento térmico, e estudar a composição química, de um OE comercial de tomilho submetido a cinco diferentes temperaturas de tratamento térmicos, no intervalo da temperatura ambiente à $100^{\circ} \mathrm{C}$, armazenados em recipientes abertos e fechados, por meio de técnicas de Análise Termogravimétrica (TG) e Calorimetria Exploratória Diferencial (DSC), para estudo do comportamento térmico e Cromatografia Gasosa (CG) para avaliação da composição química. $\mathrm{O}$ objetivo secundário proposto, foi o estudo do perfil espectroscópico vibracional deste OE, com a finalidade de examinar e comparar modificações nos os grupos vibracionais devido ao aquecimento dos OEs.

\section{MATERIAL E MÉTODOS}

\subsection{Material}

Foi utilizado o óleo essencial de tomilho (Thymus vulgaris) lote 0867 e validade 08/2020, extraído de plantas por arraste a vapor, obtido comercialmente da marca Laszlo.

\subsection{Preparação das amostras tratadas termicamente}

O experimento foi conduzido com um replicata em que foram preparados dez tratamentos, com vials contendo 1,0 $\mathrm{ml}$ de óleo essencial., sendo cinco vials fechados hermeticamente e cinco vials abertos. As amostras foram submetidas a tratamentos térmicos de $40{ }^{\circ} \mathrm{C}, 60{ }^{\circ} \mathrm{C}, 80{ }^{\circ} \mathrm{C}$ e $100{ }^{\circ} \mathrm{C}$ durante uma hora em estufa de secagem livre de circulação de ar. Após completo os tratamentos, os vials abertos foram fechados hermeticamente e todas as amostras imediatamente foram resfriadas em geladeira à $7^{\circ} \mathrm{C}$. As amostras controles utilizadas neste trabalho foram expostas em temperatura ambiente $\left(28{ }^{\circ} \mathrm{C}\right)$ armazenadas também em vials aberto e fechado. As amostras permaneceram refrigeradas até serem enviadas ao laboratório de análises térmicas da UTFPR-Campus Medianeira. As amostras foram codificadas com as legendas $\mathrm{T}$ (óleo de tomilho), aberto (A) ou fechado (F) referentes à condição de estocagem e numeradas por 1, 2, 3, 4 e 5 referentes às temperaturas ambiente $\left(28^{\circ} \mathrm{C}\right), 40^{\circ} \mathrm{C}, 60^{\circ} \mathrm{C}, 80^{\circ} \mathrm{C}$ e $100{ }^{\circ} \mathrm{C}$, respectivamente. 


\subsection{Técnicas termoanaliticas}

\subsubsection{Análise Térmica Diferencial (DTG)}

As amostras foram caracterizadas com auxílio da técnica de Análise Térmica (TG) no equipamento de análises simultânea de alta resolução (STA 6000 Simultaneous Thermal Analyzer Perkin Elmer Frontier) a temperatura controlada no intervalo entre $50^{\circ} \mathrm{C}$ a $250^{\circ} \mathrm{C}$, na taxa de aquecimento de $20{ }^{\circ} \mathrm{C}$ $\min ^{-1}$. Dez microlitros de cada amostra foram pesados no cadinho aberto de platina ródio para os

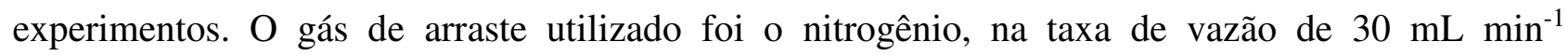
(PICOLLOTO et al., 2020).

Resultados dos testes foram expressos por meio de perfis gráficos da perda de massa decomposta durante o aquecimento em função da temperatura controlada. Em seguida, após a obtenção dos gráficos, foi aplicada a derivada de primeira ordem da Análise Termogravimétrica Diferencial (DTG), possibilitando assim caracterizar as temperaturas dos estágios de decomposição termogravimétrica para o início da reação, $T_{\text {onset, }}$ temperatura de maior cinética da decomposição termogravimétrica associada a desnaturação térmica $\left(\mathrm{T}_{\mathrm{dec}}\right)$ e temperatura de finalização da reação ( $\left.\mathrm{T}_{\text {offset}}\right)$. A temperatura $\mathrm{T}_{\mathrm{dec}}$ foi exibida nos perfis como a temperatura de inflexão.

\subsubsection{Calorimetria Exploratória Diferencial (DSC)}

Além da análise termogravimétrica, o equipamento de análises simultânea realizou a leitura do fluxo de calor entre amostra e a referência, para emitir o perfil experimental de DSC, com as mesmas condições experimentais da TG (PICOLLOTO et al., 2020).

\subsection{Caracterização química}

As análises de CG-EM foram executadas no cromatógrafo (Thermo, modelo Focus GC). As análises de cromatografia em fase gasosa acoplada à espectrometria de massas foram realizadas em equipamento da marca Thermo (modelo Focus GC), com coluna capilar DB5-MS (30 m x 0,25 mm e $0,25 \mu \mathrm{m}$ ), impacto de elétrons de $70 \mathrm{eV}$ e analisador quadrupolo linear. Foi empregado como gás de arraste o hélio com vazão na coluna de $1,0 \mathrm{mLmin}^{-1}$. A temperatura no injetor e no detector foi de $250^{\circ} \mathrm{C}$. A coluna utilizada foi uma capilar DB5-MS (30 $\mathrm{m} \times 0,25 \mathrm{~mm}$ e $0,25 \mu \mathrm{m}$ ) com a temperatura do forno ajustada a $250{ }^{\circ} \mathrm{C}$ e razão de aquecimento de $250{ }^{\circ} \mathrm{C} / \mathrm{min}$. A temperatura inicial da coluna foi de $40{ }^{\circ} \mathrm{C}$ por $2 \mathrm{~min}$, sendo programada para acréscimo de $3{ }^{\circ} \mathrm{C} \min ^{-1}$, até que atingisse a

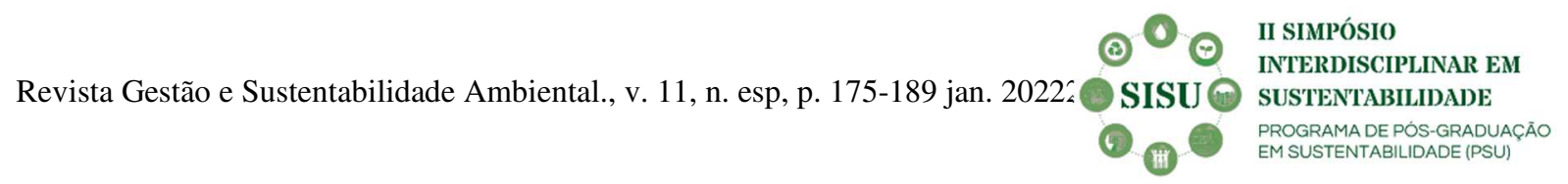




\section{GESTÃO \& SUSTENTABILIDADE AMBIENTAL \\ ১unisul Em}

temperatura máxima de $250^{\circ} \mathrm{C}$, sendo que essa temperatura foi mantida por $10 \mathrm{~min}$. Foi injetado o volume de $1 \mu \mathrm{L}$ de amostra, com modo Split 1:10. Uma mistura de alcanos lineares (C10 a C39) foi injetada no cromatográfico nas mesmas condições usadas com padrão para os cálculos do índice de retenção com programação linear de temperatura. Comparou-se o índice de retenção dos resultados identificados com as bibliotecas disponíveis na literatura (ADAMS, 2017; NISTBOOK, 2020).

\subsection{Espectroscopia de Infravermelho por Transformada de Fourier (FTIR)}

A Espectroscopia de Infravermelho utilizando a transformada de Fourier (FTIR) com reflectância total atenuada (ATR) foi realizada no espectrômetro de infravermelho (modelo Cary 630 FTIR, marca Agilent Technologies). O intervalo espectral foi de 4000 a $400 \mathrm{~cm}^{-1}$, varredura de 64 scans e $4 \mathrm{~cm}^{-1}$ de precisão (PICOLLOTO et al., 2020). As análises foram realizadas no Laboratório de Espectroscopia do LACE - Laboratório de Análises Cromatográficas e Espectroscópicas do Instituto Federal do Campus de Umuarama-PR.

\section{RESULTADOS E DISCUSSÃO}

\subsection{Técnicas termoanaliticas}

Inicialmente foi realizada uma varredura dinâmica TG-DTG do óleo essencial de tomilho no intervalo de $25^{\circ} \mathrm{C}$ a $250^{\circ} \mathrm{C}$, para que o comportamento térmico fosse avaliado. Na Figura 1 estão as curvas experimentais para os tratamentos controle, armazenados em vials aberto e fechado, como exemplo.

Figura 1 - Perfis experimentais TG e DTG. (a) óleo essencial de tomilho em vial aberto submetido à temperatura ambiente (controle). (b) óleo essencial de tomilho em vial fechado submetido à temperatura ambiente (controle).
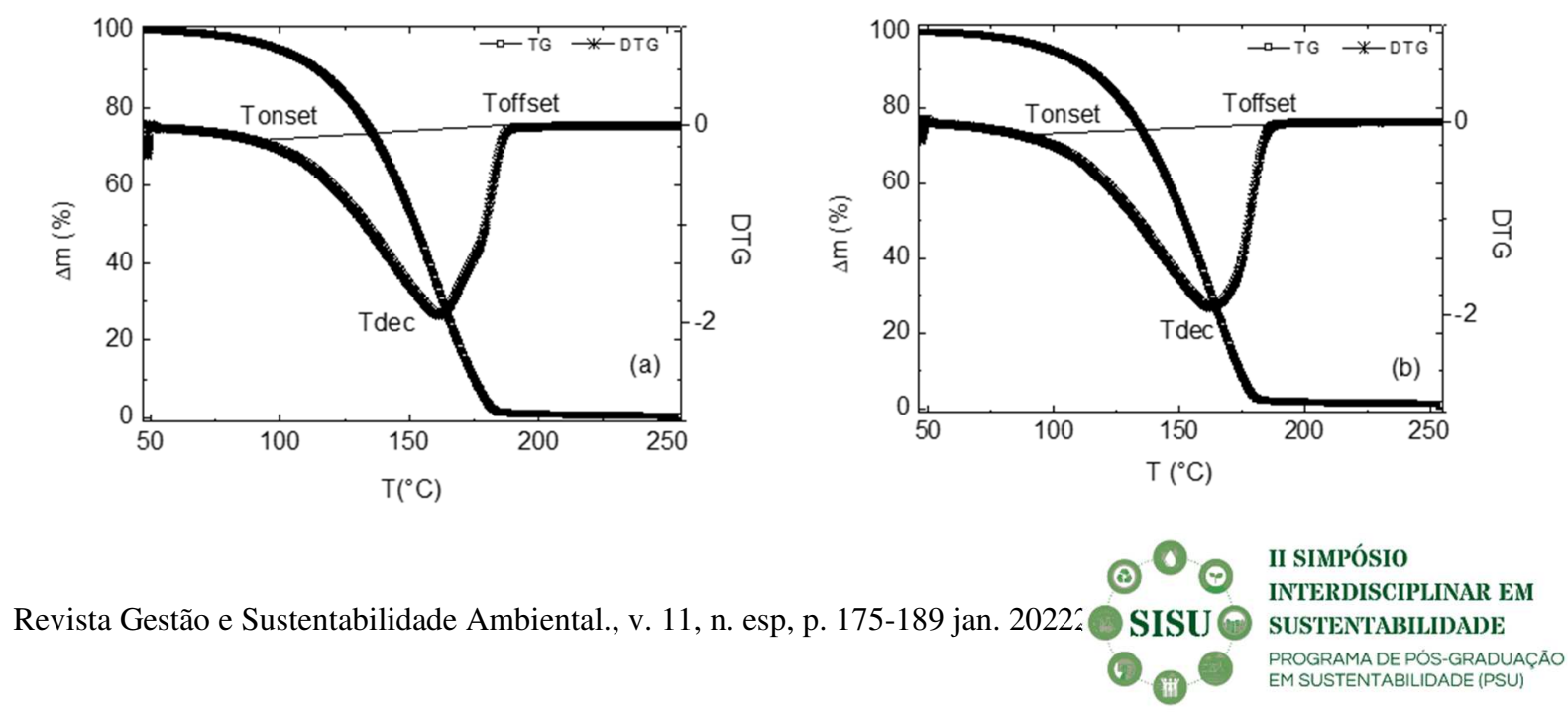


\section{GESTÃO \& SUSTENTABILIDADE AMBIENTAL}

\section{sunisul sim}

Por meio da curva TG observou-se que a decomposição termogravimétrica que pode ser associada ao fenômeno de vaporização. A perda de massa sugere que não houve oxidação no óleo essencial de tomilho, apenas vaporização dos compostos voláteis e aquosos com o aquecimento. Nas curvas derivadas DTG, pode-ses observar as temperaturas de início da termodegradação ( $\left.T_{\text {onset}}\right)$ que é vinculada a estabilidade térmica pois define o início da vaporização dos $\mathrm{OE}$. $\mathrm{T}_{\mathrm{dec}}$ define a temperatura de maior cinética de decomposição e $\mathrm{T}_{\text {offset }}$ registra o final da decomposição. A Tabela 1 apresenta os resultados das temperaturas do comportamento térmico do óleo essencial de tomilho, acondicionado em vials abertos e fechados.

Tabela 1 - Temperaturas típicas obtidas por DTG e DSC para o óleo essencial de tomilho em vials aberto (TA) e fechado (TF) tratados termicamente.

\begin{tabular}{|c|c|c|c|c|}
\hline Tratamentos & $\mathbf{T}_{\text {onset }}\left({ }^{\circ} \mathbf{C}\right)$ & $\mathbf{T}_{\text {dec }}\left({ }^{\circ} \mathbf{C}\right)$ & $\mathbf{T}_{\text {offset }}\left({ }^{\circ} \mathbf{C}\right)$ & $\mathbf{T}_{\text {vap }}\left({ }^{\circ} \mathbf{C}\right)$ \\
\hline TA1 (controle $28^{\circ} \mathrm{C}$ ) & $79,5 \pm 0,3$ & $170,9 \pm 0,3$ & $188,3 \pm 0,3$ & $167,7 \pm 0,3$ \\
\hline TA2 $\left(40^{\circ} \mathrm{C}\right)$ & $75,2 \pm 0,3$ & $170,7 \pm 0,3$ & $194,1 \pm 0,3$ & $172,6 \pm 0,3$ \\
\hline TA3 $\left(60^{\circ} \mathrm{C}\right)$ & $77,1 \pm 0,3$ & $167,6 \pm 0,3$ & $192,2 \pm 0,3$ & $171,9 \pm 0,3$ \\
\hline TA4 $\left(80^{\circ} \mathrm{C}\right)$ & $69,9 \pm 0,3$ & $159,8 \pm 0,3$ & $186,4 \pm 0,3$ & $161,5 \pm 0,3$ \\
\hline TA5 $\left(100^{\circ} \mathrm{C}\right)$ & $69,1 \pm 0,3$ & $161,5 \pm 0,3$ & $187,2 \pm 0,3$ & $164,4 \pm 0,3$ \\
\hline $\mathrm{TF} 1$ (controle $28^{\circ} \mathrm{C}$ ) & $76,2 \pm 0,3$ & $162,2 \pm 0,3$ & $185,8 \pm 0,3$ & $165,5 \pm 0,3$ \\
\hline $\mathrm{TF} 2\left(40^{\circ} \mathrm{C}\right)$ & $74,4 \pm 0,3$ & $164,7 \pm 0,3$ & $188,8 \pm 0,3$ & $168,5 \pm 0,3$ \\
\hline $\mathrm{TF} 3\left(60^{\circ} \mathrm{C}\right)$ & $76,4 \pm 0,3$ & $166,9 \pm 0,3$ & $190,8 \pm 0,3$ & $169,1 \pm 0,3$ \\
\hline $\mathrm{TF} 4\left(80^{\circ} \mathrm{C}\right)$ & $76,7 \pm 0,3$ & $162,7 \pm 0,3$ & $187,5 \pm 0,3$ & $165,4 \pm 0,3$ \\
\hline TF5 $\left(100^{\circ} \mathrm{C}\right)$ & $77,3 \pm 0,3$ & $167,1 \pm 0,3$ & $189,9 \pm 0,3$ & $167,7 \pm 0,3$ \\
\hline
\end{tabular}

O exame das análises DTG confirmaram que houve um único estágio de decomposição termogravimétrica para cada modelo experimental. No entanto, ao comparar os resultados do óleo essencial de tomilho acondicionado em recipiente aberto, observou-se uma maior flutuação das temperaturas no óleo essencial tratado a diferentes temperaturas. $\mathrm{O}$ tratamento controle (temperatura ambiente - amostra TA1) revelou-se mais resistente termicamente à decomposição em relação as demais temperaturas de tratamento. Nos tratamentos a 40 e $60{ }^{\circ} \mathrm{C}$ a $\mathrm{T}_{\text {onset }}$ esteve estabelecida cerca de 2 a $4^{\circ} \mathrm{C}$, inferior ao tratamento controle. E essa diferença se intensificou a aproximadamente $10{ }^{\circ} \mathrm{C}$ nas amostras submetidas às temperaturas de 80 e $100{ }^{\circ} \mathrm{C}$. Esse resultado não se revelou muito

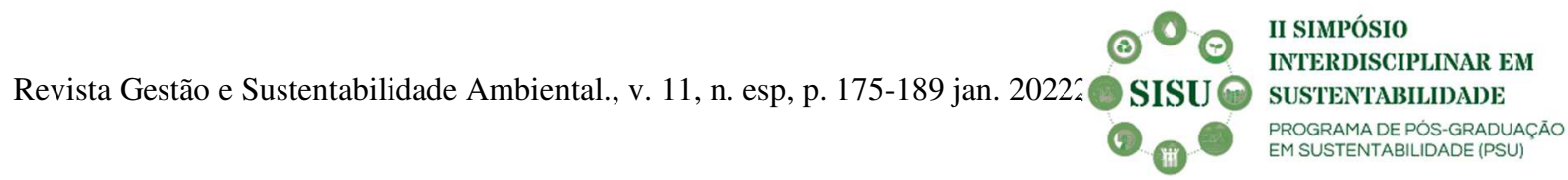




\section{GESTÃO \& SUSTENTABILIDADE AMBIENTAL}

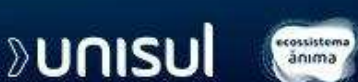

satisfatório para o tratamento térmico nos tratamentos de óleo essencial de tomilho armazenados em recipientes abertos, sugerindo que entre a temperatura ambiente $\left(28^{\circ} \mathrm{C}\right)$ até $60^{\circ} \mathrm{C}$, esse óleo essencial suportou maior estabilidade térmica.

No entanto, para cada tratamento acondicionado hermeticamente os resultados apontaram excelente estabilidade térmica nos óleos essenciais tratados até $100{ }^{\circ} \mathrm{C}$, sendo apreciada insignificante flutuação da temperatura. No óleo essencial de tomilho aquecido à $60^{\circ} \mathrm{C}$ a temperatura de inflexão foi levemente superior as demais. Porém, a $T_{\text {dec }}$ obtida com auxílio da DTG coincidiu aproximadamente com a temperatura de pico do perfil DSC, cuja concavidade orientada para cima mostrou que a transição de fase foi endotérmica. Para os óleos essenciais esse fenômeno evidencia a sua vaporização, por meio da transição entre os estados líquido e gasoso (IONASHIRO, GIOLITO, 2004). Na Figura 2 estão as curvas DSC para os tratamentos controle, armazenados em vials aberto e fechado, como exemplo.

Figura 2 - Perfis experimentais DSC. (a) óleo essencial de tomilho em vial aberto submetido à temperatura ambiente (controle). (b) óleo essencial de tomilho em vial fechado submetido à temperatura ambiente (controle).
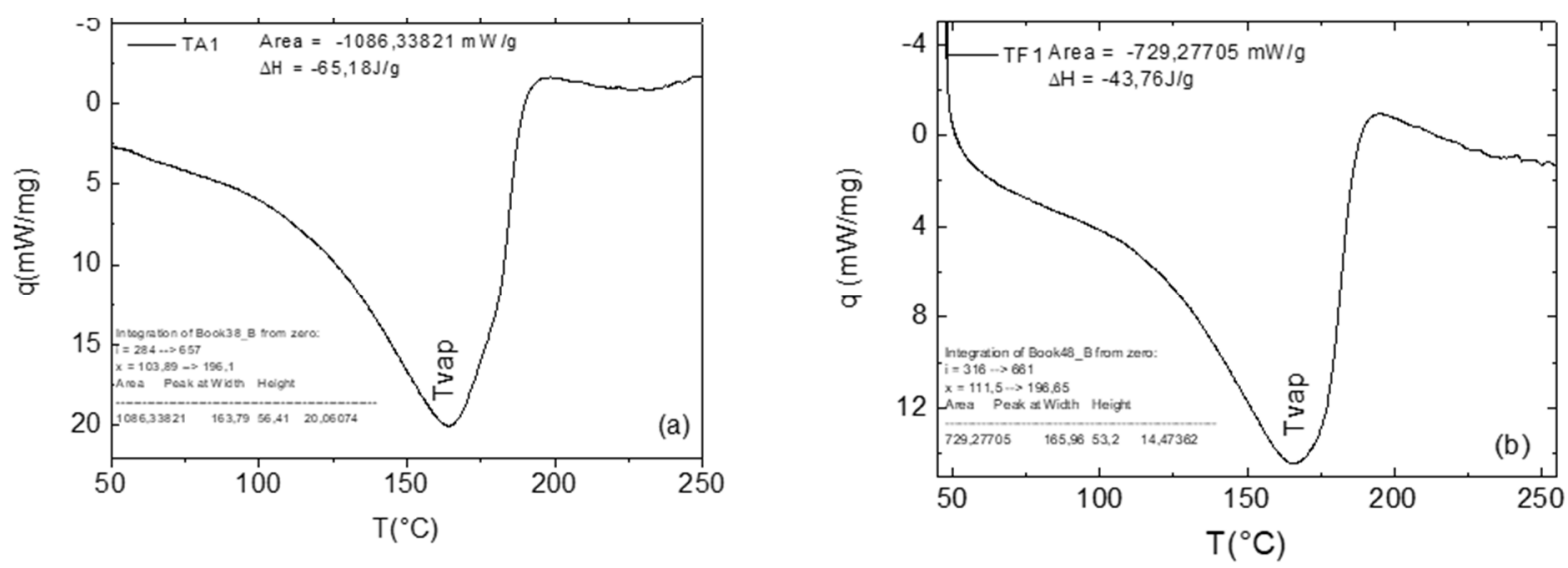

Por meio do sinal resultante, a curva característica do fluxo de calor, (q) medido em $\mathrm{mW} \mathrm{mg}^{-1}$, em função da temperatura foi encontrada. Durante o aquecimento, as transições de fase de primeira ordem foram observadas nos perfis analisando-se as concavidades dos picos.

A temperatura de vaporização resultante nos picos DTG foi associada ao pico endotérmico do perfil DSC (representado pela concavidade orientada para cima). A variação da entalpia da transição $(\Delta \mathrm{H})$ física foi calculada por meio da integral da área do pico característico, pois o sinal negativo foi associado ao fenômeno endotérmico.

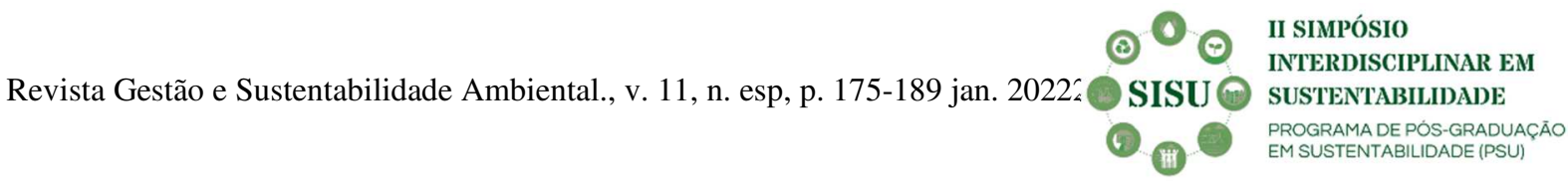




\section{sunisul}

Fisicamente, a variação da entalpia é o calor, a pressão constante, necessário para que haja transição de fase dos estados líquido e gasoso e depende diretamente da variação de temperatura absorvida no sistema. A maioria das reações que envolvem vaporização acontecem naturalmente. Com a evolução do fenômeno, a energia é absorvida pelos compostos permanentes (CANEVAROLO, 2017).

De acordo com os resultados, pode-se observar a intensificação do módulo da variação da entalpia com o aumento da temperatura de tratamento térmico do óleo essencial de tomilho. Para os tratamentos com acondicionamento aberto, a variação da entalpia entre a amostra tratada a $100{ }^{\circ} \mathrm{C}$ a controle se intensificou 10,5 vezes, enquanto que nas amostras acondicionadas hermeticamente, a razão da variação da entalpia foi de aproximadamente 14,8 vezes. Esse resultado inferiu que o tratamento pelo calor demandou maior energia térmica durante a transição de fase. Ao aumentar a temperatura de tratamento das amostras, a entalpia foi substancialmente ampliada, indicando que durante a transição de fase, maior energia foi necessária para vaporizar o óleo essencial e aumento significativo do calor latente.

Logo, os resultados obtidos por meio das técnicas termogravimétricas indicaram que para o óleo essencial de tomilho a aplicação de calor durante não alterou significativamente suas temperaturas características de vaporização ou estabilidade térmica, no entanto, elevaram significativamente a energia térmica dos compostos para que houvesse a transição de fase. Outro resultado relevante indicado foi a estabilidade das temperaturas nas amostras acondicionadas hermeticamente, sugerindo ser um método seguro de estocagem para armazenar o óleo essencial de tomilho. Os resultados termoanalíticos encontrados para os tratamentos abertos aquecidos a 80 e $100{ }^{\circ} \mathrm{C}$ apresentaram estabilidade térmica inferior, ao comparar com o óleo essencial tratado até $60{ }^{\circ} \mathrm{C}$. Na prática, de acordo com esses resultados, pode não convir aplicar calor em produtos contendo o óleo essencial de tomilho em que sejam empregadas temperaturas superiores a $80^{\circ} \mathrm{C}$ no processo e em sistema aberto, para manter a estabilidade térmica do óleo essencial e evitar a vaporização dos seus compostos majoritários.

\subsection{Caracterização química}

No óleo essencial de tomilho foram identificados 46 compostos, em que o linalool (32,6\%), tymol $(12,6 \%)$ e cis-geraniol $(8,7 \%)$ foram os componentes majoritários (Figura 3). Os compostos foram agrupados de acordo com as classes químicas. A composição química apresentou 39\% de compostos

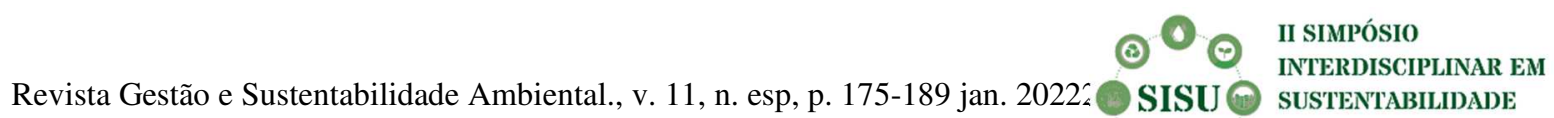




\section{GESTÃO \& SUSTENTABILIDADE AMBIENTAL \\ ¿unisul}

sesquiterpênicos, 38\% de monoterpênicos oxigenados, $19 \%$ de monoterpênicos e 0,74\% de sesquiterpênicos oxigenados. Houve discreta diminuição e aumento de monoterpênicos para os tratamentos em sistema aberto e fechado, respectivamente. Os monoterpênicos oxigenados diminuíram nos sistemas abertos e mantiveram a estabilidade nos sistemas fechados, conforme o aumento da temperatura nos tratamentos térmicos. Já os sesquiterpênicos, aumentaram nos sistemas abertos e se mantiveram estáveis nos sistemas fechados. Em contrapartida, foi observada a diminuição dos compostos sesquiterpênicos oxigenados nos sistemas abertos e estabilidade nos sistemas fechados (Tabela 2).

Figura 3 - Estruturas químicas dos componentes majoritários do óleo essencial de tomilho.

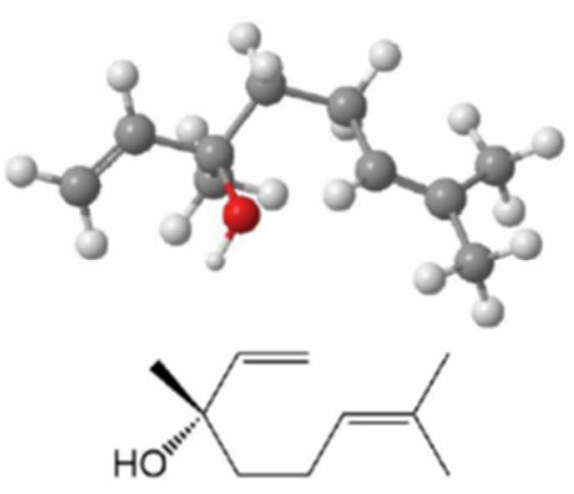

Linalool

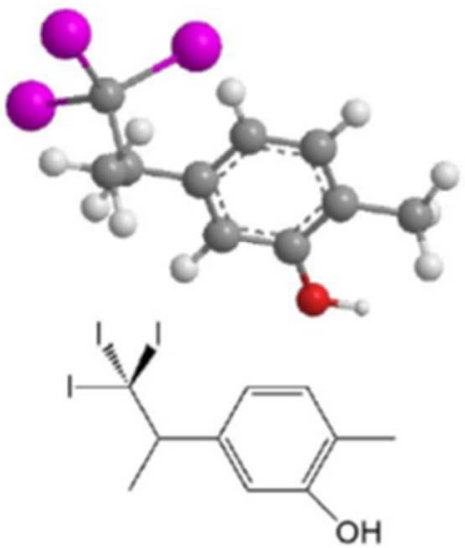

Tymol

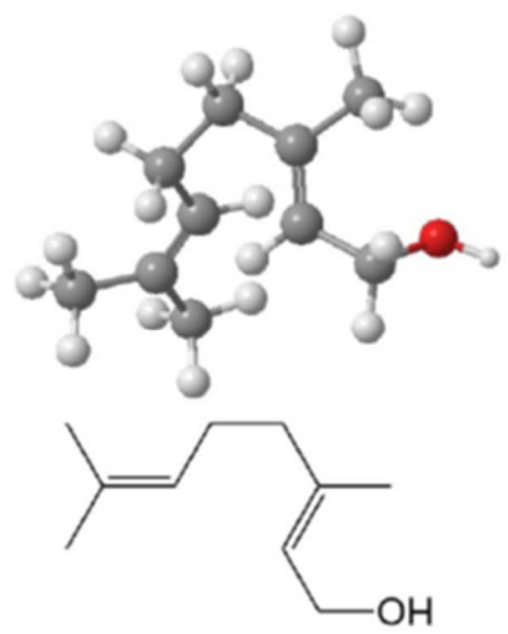

Cis-geraniol

Tabela 2 - Classes químicas do óleo essencial de tomilho (T) a temperatura ambiente (1) e aquecido a 40 (2), 60 (3), 80 (4) e $100(5)^{\circ} \mathrm{C}$, em sistema aberto (A) e fechado (B).

\begin{tabular}{l|rrrrr|rrrrr}
\hline Classes químicas & $\begin{array}{r}\text { TA1 } \\
\mathbf{( \% )}\end{array}$ & $\begin{array}{r}\text { TA2 } \\
(\boldsymbol{\%})\end{array}$ & $\begin{array}{r}\text { TA3 } \\
(\boldsymbol{\%})\end{array}$ & $\begin{array}{r}\text { TA4 } \\
(\mathbf{\%})\end{array}$ & $\begin{array}{r}\text { TA5 } \\
\mathbf{( \% )}\end{array}$ & $\begin{array}{r}\text { TF1 } \\
(\boldsymbol{\%})\end{array}$ & $\begin{array}{r}\text { TF2 } \\
(\boldsymbol{\%})\end{array}$ & $\begin{array}{r}\text { TF3 } \\
(\boldsymbol{\%})\end{array}$ & $\begin{array}{r}\text { TF4 } \\
(\boldsymbol{\%})\end{array}$ & $\begin{array}{r}\text { TF5 } \\
(\boldsymbol{\%})\end{array}$ \\
\hline Monoterpeno & 16,67 & 17,68 & 15,48 & 18,05 & 7,83 & 15,91 & 38,11 & 14,32 & 14,01 & 17,06 \\
Monoterpeno oxigenado & 44,15 & 42,08 & 43,81 & 42,00 & 22,35 & 44,73 & 9,05 & 43,87 & 44,87 & 43,7 \\
Sesquiterpeno & 38,09 & 39,34 & 37,76 & 38,29 & 42,14 & 38,16 & 37,43 & 40,35 & 39,13 & 38,02 \\
Sesquiterpeno oxigenado & 1,02 & 0,66 & 0,99 & 0,54 & 0,94 & 0,86 & 0,14 & 0,39 & 1,02 & 0,82 \\
Total & $\mathbf{9 9 , 9 3}$ & $\mathbf{9 9 , 7 6}$ & $\mathbf{9 8 , 0 4}$ & $\mathbf{9 8 , 8 8}$ & $\mathbf{7 3 , 2 6}$ & $\mathbf{9 9 , 6 6}$ & $\mathbf{8 4 , 7 3}$ & $\mathbf{9 8 , 9 3}$ & $\mathbf{9 9 , 0 3}$ & $\mathbf{9 9 , 6}$ \\
\hline
\end{tabular}




\section{GESTÃO \& SUSTENTABILIDADE AMBIENTAL \\ ১unisul sims}

Os principais compostos identificados no óleo essencial de tomilho mostraram um alto teor de monoterpenos (aproximadamente 80\%) e menor teor de sesquiterpenos para as amostras controle, acondicionadas de formas distintas. Observou-se que com o aumento da temperatura aplicada nos tratamentos houve uma discreta redução da concentração dos monoterpenos no óleo essencial, porém, a composição dos sesquiterpenos oxigenados flutuaram de acordo com o sistema de armazenamento, sendo que as quantidades desses compostos apresentaram estabilidade nos vials fechados e uma tendência de redução da concentração de sesquiterpenos com o aumento da temperatura nos vials abertos.

\subsection{Espectroscopia de Infravermelho por Transformada de Fourier (FTIR)}

A Espectroscopia Infravermelha por Transformada de Fourier é uma das técnicas mais amplamente utilizadas para identificação dos grupos funcionais e tem o objetivo de encontrar os espectros de um grande número de compostos orgânicos nos estados líquido, sólido e vapor. A absorção infravermelha é observada quando a frequência de radiação incidente está em ressonância com a frequência de vibração da molécula; e houver alteração do momento de dipolo elétrico (MAIER, 2004).

Os espectrogramas foram obtidos entre $3500 \mathrm{~cm}^{-1}$ a $500 \mathrm{~cm}^{-1}$. Nas Tabelas 3 e 4 foram identificadas bandas dos halogêneos com estiramento C-X $550 \mathrm{~cm}^{-1}, 600 \mathrm{~cm}^{-1}, 700 \mathrm{~cm}^{-1,} 740 \mathrm{~cm}^{-1}$, $1000 \mathrm{~cm}^{-1} \mathrm{e} 1400 \mathrm{~cm}^{-1}$, na banda de deformação fora do planos $\mathrm{R}_{2} \mathrm{C}=\mathrm{CHR} 800$, nos éteres a banda de estiramento C-O 1100, nos aromáticos a banda de ligação C=C 1500, no anel aromático a banda 940 $\mathrm{cm}^{-1}$ de estiramento e ligação com hidrogênio isolado em benzeno, nos éteres as bandas com estiramento C-O 1100 e 1160, nos ésteres as bandas de estiramento C-O 1250 e 1300, nos aromáticos as bandas com estiramento $\mathrm{C}=\mathrm{C} 1500$ e 1600, nas ligações alifáticas as bandas com estiramento $\mathrm{C}-\mathrm{H}$ $2840 \mathrm{~cm}^{-1}$ e $2920 \mathrm{~cm}^{-1}$, a banda O-H quelato com estiramento $\mathrm{C}=\mathrm{O}, \mathrm{NO}_{2}, 2945 \mathrm{~cm}^{-1}$ e o grupo das aminas aromáticas com estiramento $\mathrm{N}-\mathrm{H} 3400 \mathrm{~cm}^{-1}$, permaneceram estáveis termoquimicamente, pois não foram observadas flutuações de bandas apesar do tratamento térmico. 


\section{GESTÃO \& SUSTENTABILIDADE AMBIENTAL}

\section{ounisul}

Tabela 3 - Bandas de absorção e compostos identificados do óleo essencial de tomilho acondicionado em vials abertos.

\begin{tabular}{|c|c|c|c|c|c|c|c|c|}
\hline $\begin{array}{c}\text { Número de } \\
\text { onda } \\
\text { central } \\
\left(\mathrm{cm}^{-1}\right)\end{array}$ & Tipo de composto & Grupo funcional & $\begin{array}{l}\text { Modo de } \\
\text { vibração }\end{array}$ & TA1 & TA2 & TA3 & TA4 & TA5 \\
\hline 550 & $\mathrm{C}-\mathrm{Br}$ & C-X (X=Halogênio) & Estiramento & $\mathrm{X}$ & $\mathrm{X}$ & $\mathrm{X}$ & $\mathrm{X}$ & $\mathrm{X}$ \\
\hline 550 & $\mathrm{C}-\mathrm{Br}$ & C-X (X=Halogênio) & Estiramento & $\mathrm{X}$ & $\mathrm{X}$ & $\mathrm{X}$ & $\mathrm{X}$ & $\mathrm{X}$ \\
\hline 600 & $\mathrm{C}-\mathrm{Cl}$ & C-X (X=Halogênio) & Estiramento & $\mathrm{X}$ & $\mathrm{X}$ & $\mathrm{X}$ & $\mathrm{X}$ & $\mathrm{X}$ \\
\hline 630 & $\mathrm{C}-\mathrm{Br}$ & C-X (X=Halogênio) & Estiramento & & & & & \\
\hline 650 & $\mathrm{C}-\mathrm{Br}$ & C-X (X=Halogênio) & Estiramento & $\mathrm{X}$ & & & $\mathrm{X}$ & \\
\hline 680 & $\mathrm{C}-\mathrm{Br}$ & C-X (X=Halogênio) & Estiramento & & & $\mathrm{X}$ & & \\
\hline 700 & $\mathrm{C}-\mathrm{Cl}$ & C-X (X=Halogênio) & Estiramento & $\mathrm{X}$ & $\mathrm{X}$ & $\mathrm{X}$ & $\mathrm{X}$ & $\mathrm{X}$ \\
\hline 720 & $\mathrm{C}-\mathrm{Cl}$ & C-X (X=Halogênio) & Estiramento & & & & & \\
\hline 740 & $\mathrm{C}-\mathrm{Cl}$ & C-X (X=Halogênio) & Estiramento & $\mathrm{X}$ & $\mathrm{X}$ & & $\mathrm{X}$ & $\mathrm{X}$ \\
\hline 800 & & $\mathrm{R}_{2} \mathrm{C}=\mathrm{CHR}$ & $\begin{array}{l}\text { C-H fora do } \\
\text { plano }\end{array}$ & $\mathrm{X}$ & $\mathrm{X}$ & $\mathrm{X}$ & $\mathrm{X}$ & \\
\hline 920 & $\mathrm{O}-\mathrm{H}$ & & $\begin{array}{l}\text { Deformação } \\
\text { angula fora do } \\
\text { plano }\end{array}$ & $\mathrm{X}$ & $\mathrm{X}$ & $\mathrm{X}$ & $\mathrm{X}$ & \\
\hline 1000 & $\mathrm{C}-\mathrm{F}$ & C-X (X=Halogênio) & Estiramento & $X$ & $\mathrm{X}$ & $X$ & $\mathrm{X}$ & $\mathrm{X}$ \\
\hline 1100 & Éteres alifáticos & $\mathrm{C}-\mathrm{O}$ de éteres & Estiramento & $\mathrm{X}$ & $\mathrm{X}$ & $\mathrm{X}$ & & $\mathrm{X}$ \\
\hline 1210 & $\begin{array}{l}\text { Ésteres insaturados e } \\
\text { aromáticos: } 2 \text { bandas }\end{array}$ & C-O de álcoois ésteres & Estiramento & & $\mathrm{X}$ & & & $\mathrm{X}$ \\
\hline 1220 & $\begin{array}{l}\text { Ésteres insaturados e } \\
\text { aromáticos: } 2 \text { bandas }\end{array}$ & C-O de álcoois ésteres & Estiramento & $\mathrm{X}$ & & & $\mathrm{X}$ & \\
\hline 1250 & $\begin{array}{l}\text { Ésteres insaturados e } \\
\text { aromáticos: } 2 \text { bandas }\end{array}$ & C-O de álcoois ésteres & Estiramento & & $\mathrm{X}$ & $\mathrm{X}$ & $\mathrm{X}$ & \\
\hline 1280 & $\begin{array}{l}\text { Ésteres insaturados e } \\
\text { aromáticos: } 2 \text { bandas }\end{array}$ & C-O de álcoois ésteres & Estiramento & $\mathrm{X}$ & & $\mathrm{X}$ & $\mathrm{X}$ & $\mathrm{X}$ \\
\hline 1380 & C-F & C-X (X=Halogênio) & Estiramento & & $\mathrm{X}$ & $\mathrm{X}$ & & $\mathrm{X}$ \\
\hline 1400 & $\mathrm{C}-\mathrm{F}$ & C-X (X=Halogênio) & Estiramento & $\mathrm{X}$ & $\mathrm{X}$ & $\mathrm{X}$ & $\mathrm{X}$ & $\mathrm{X}$ \\
\hline 1430 & & & & $\mathrm{X}$ & & $\mathrm{X}$ & & \\
\hline 1440 & & & & $\mathrm{X}$ & & & $\mathrm{X}$ & \\
\hline 1500 & & $\mathrm{C}=\mathrm{C}$ aromáticos & Estiramento & $\mathrm{X}$ & $\mathrm{X}$ & $\mathrm{X}$ & & $\mathrm{X}$ \\
\hline 1580 & $\mathrm{NH}_{2}$ & & $\begin{array}{c}\text { Deformação } \\
\text { angula simétrica } \\
\text { no plano }\end{array}$ & $\mathrm{X}$ & & & $\mathrm{X}$ & \\
\hline 1630 & $\begin{array}{l}\text { Amidas N- } \\
\text { substituídas }\end{array}$ & $\mathrm{C}=\mathrm{O}$ de amidas & Banda única & $\mathrm{X}$ & $\mathrm{X}$ & $\mathrm{X}$ & & $\mathrm{X}$ \\
\hline 2840 & Carbono terciário & C-H alifáticos & Estiramento & $\mathrm{X}$ & $\mathrm{X}$ & $\mathrm{X}$ & $\mathrm{X}$ & $\mathrm{X}$ \\
\hline 2920 & Carbono terciário & C-H alifáticos & Estiramento & $\mathrm{x}$ & $\mathrm{X}$ & $\mathrm{X}$ & $\mathrm{X}$ & $\mathrm{X}$ \\
\hline 2945 & $\mathrm{C}=\mathrm{O}, \mathrm{NO}_{2}$ & O-H (quelato) & Estiramento & $\mathrm{X}$ & $\mathrm{X}$ & $\mathrm{X}$ & $\mathrm{X}$ & $\mathrm{X}$ \\
\hline 3400 & aminas aromáticas & $\mathrm{N}-\mathrm{H}$ & Estiramento & $\mathrm{X}$ & $X$ & $X$ & $X$ & $X$ \\
\hline
\end{tabular}




\section{GESTÃO \& SUSTENTABILIDADE AMBIENTAL}

\section{ounisul}

Tabela 4 - Bandas de absorção e compostos identificados do óleo essencial de tomilho acondicionado em vials fechados.

\begin{tabular}{|c|c|c|c|c|c|c|c|c|}
\hline $\begin{array}{c}\text { Número de } \\
\text { onda } \\
\text { central } \\
\left(\mathrm{cm}^{-1}\right) \\
\end{array}$ & Tipo de composto & Grupo funcional & $\begin{array}{l}\text { Modo de } \\
\text { vibração }\end{array}$ & TF1 & TF2 & TF3 & TF4 & TF5 \\
\hline 550 & $\mathrm{C}-\mathrm{Br}$ & C-X (X=Halogênio) & Estiramento & $X$ & $\mathrm{X}$ & $X$ & $\mathrm{X}$ & $X$ \\
\hline 550 & $\mathrm{C}-\mathrm{Br}$ & C-X (X=Halogênio) & Estiramento & $\mathrm{X}$ & $\mathrm{X}$ & $\mathrm{X}$ & $\mathrm{X}$ & $\mathrm{X}$ \\
\hline 600 & $\mathrm{C}-\mathrm{Cl}$ & C-X (X=Halogênio) & Estiramento & $\mathrm{X}$ & $\mathrm{X}$ & $X$ & $\mathrm{X}$ & $\mathrm{X}$ \\
\hline 630 & $\mathrm{C}-\mathrm{Br}$ & C-X (X=Halogênio) & Estiramento & & & $\mathrm{X}$ & $\mathrm{X}$ & $\mathrm{X}$ \\
\hline 650 & $\mathrm{C}-\mathrm{Br}$ & C-X (X=Halogênio) & Estiramento & $\mathrm{X}$ & & & & \\
\hline 680 & $\mathrm{C}-\mathrm{Br}$ & C-X (X=Halogênio) & Estiramento & & & $\mathrm{X}$ & & \\
\hline 700 & $\mathrm{C}-\mathrm{Cl}$ & C-X (X=Halogênio) & Estiramento & $\mathrm{X}$ & $\mathrm{X}$ & $\mathrm{X}$ & $\mathrm{X}$ & $\mathrm{X}$ \\
\hline 720 & $\mathrm{C}-\mathrm{Cl}$ & C-X (X=Halogênio) & Estiramento & & & & $\mathrm{X}$ & \\
\hline 740 & $\mathrm{C}-\mathrm{Cl}$ & C-X (X=Halogênio) & Estiramento & $\mathrm{X}$ & $\mathrm{X}$ & $X$ & & $\mathrm{X}$ \\
\hline 800 & & $\mathrm{R}_{2} \mathrm{C}=\mathrm{CHR}$ & $\begin{array}{l}\mathrm{C}-\mathrm{H} \text { fora do } \\
\text { plano }\end{array}$ & $\mathrm{X}$ & $\mathrm{X}$ & $\mathrm{X}$ & $\mathrm{X}$ & $\mathrm{X}$ \\
\hline 920 & $\mathrm{O}-\mathrm{H}$ & & $\begin{array}{l}\text { Deformação } \\
\text { angula fora do } \\
\text { plano }\end{array}$ & $\mathrm{X}$ & $\mathrm{X}$ & $\mathrm{X}$ & & \\
\hline 1000 & $\mathrm{C}-\mathrm{F}$ & C-X (X=Halogênio) & Estiramento & $\mathrm{X}$ & $\mathrm{X}$ & $\mathrm{X}$ & $\mathrm{X}$ & $\mathrm{X}$ \\
\hline 1100 & Éteres alifáticos & C-O de éteres & Estiramento & $\mathrm{X}$ & $\mathrm{X}$ & $\mathrm{X}$ & $\mathrm{X}$ & $\mathrm{X}$ \\
\hline 1210 & $\begin{array}{l}\text { Ésteres insaturados e } \\
\text { aromáticos: } 2 \text { bandas }\end{array}$ & C-O de álcoois ésteres & Estiramento & & & & & $\mathrm{X}$ \\
\hline 1220 & $\begin{array}{l}\text { Ésteres insaturados e } \\
\text { aromáticos: } 2 \text { bandas }\end{array}$ & C-O de álcoois ésteres & Estiramento & $\mathrm{X}$ & & $\mathrm{X}$ & & \\
\hline 1250 & $\begin{array}{l}\text { Ésteres insaturados e } \\
\text { aromáticos: } 2 \text { bandas }\end{array}$ & C-O de álcoois ésteres & Estiramento & & $\mathrm{X}$ & & & \\
\hline 1280 & $\begin{array}{l}\text { Ésteres insaturados e } \\
\text { aromáticos: } 2 \text { bandas }\end{array}$ & C-O de álcoois ésteres & Estiramento & $\mathrm{X}$ & $\mathrm{X}$ & $\mathrm{X}$ & & $\mathrm{X}$ \\
\hline 1380 & C-F & C-X (X=Halogênio) & Estiramento & & $\mathrm{X}$ & & & $\mathrm{X}$ \\
\hline 1400 & C-F & C-X (X=Halogênio) & Estiramento & $\mathrm{X}$ & $\mathrm{X}$ & $\mathrm{X}$ & $\mathrm{X}$ & $\mathrm{X}$ \\
\hline 1430 & & & & $\mathrm{X}$ & & & & $\mathrm{X}$ \\
\hline 1440 & & & & $\mathrm{X}$ & $\mathrm{X}$ & $\mathrm{X}$ & & \\
\hline 1500 & & $\mathrm{C}=\mathrm{C}$ aromáticos & Estiramento & $\mathrm{X}$ & $\mathrm{X}$ & $\mathrm{X}$ & $\mathrm{X}$ & $\mathrm{X}$ \\
\hline 1580 & $\mathrm{NH}_{2}$ & & $\begin{array}{c}\text { Deformação } \\
\text { angula simétrica } \\
\text { no plano }\end{array}$ & $\mathrm{X}$ & & $\mathrm{X}$ & & \\
\hline 1630 & $\begin{array}{l}\text { Amidas N- } \\
\text { substituídas }\end{array}$ & $\mathrm{C}=\mathrm{O}$ de amidas & Banda única & $\mathrm{X}$ & $\mathrm{X}$ & & & $\mathrm{X}$ \\
\hline 2840 & Carbono terciário & $\mathrm{C}-\mathrm{H}$ alifáticos & Estiramento & $\mathrm{X}$ & $\mathrm{X}$ & $\mathrm{X}$ & $\mathrm{X}$ & $\mathrm{X}$ \\
\hline 2920 & Carbono terciário & C-H alifáticos & Estiramento & $\mathrm{X}$ & $\mathrm{X}$ & $\mathrm{X}$ & $\mathrm{X}$ & $\mathrm{X}$ \\
\hline 2945 & $\mathrm{C}=\mathrm{O}, \mathrm{NO}_{2}$ & $\mathrm{O}-\mathrm{H}$ (quelato) & Estiramento & $\mathrm{X}$ & $\mathrm{X}$ & $\mathrm{X}$ & $\mathrm{X}$ & $\mathrm{X}$ \\
\hline 3400 & aminas aromáticas & N-H & Estiramento & $X$ & $X$ & $X$ & $X$ & $X$ \\
\hline
\end{tabular}


A banda com deformação angular fora do plano com ligação $\mathrm{O}-\mathrm{H} 920 \mathrm{~cm}^{-1}$ apresentou estabilidade até $80{ }^{\circ} \mathrm{C}$ em sistema aberto e até $60^{\circ} \mathrm{C}$ em sistema fechado indicando a presença de anéis aromáticos em sua composição. As bandas $650 \mathrm{~cm}^{-1}, 1220 \mathrm{~cm}^{-1}, 1430 \mathrm{~cm}^{-1}, 1440 \mathrm{~cm}^{-1}$ e $1580 \mathrm{~cm}^{-1}$ foram detectadas nos tratamentos controles e flutuaram entre ausência e presença nos demais tratamentos submetidos ao calor, tanto em sistema aberto quanto fechado, indicando reações reversíveis de degradação e síntese para seus grupos funcionais que representam.

As bandas $630 \mathrm{~cm}^{-1}$ e $1380 \mathrm{~cm}^{-1}$ do grupo dos halogêneos e $1210 \mathrm{~cm}^{-1}$ dos grupos dos álcoois ésteres saturados foram em captadas nos tratamentos com aplicação de calor, no entanto não foram observadas no tratamento controle, sugerindo o aquecimento como fator impulsionador para a formação de compostos contendo grupo funcional de halogênio.

Os resultados indicaram que a aplicação do calor e estocagem do OE influi na estabilidade ou constituição de compostos químicos com diferentes funções orgânicas, possivelmente tendo o calor como agente catalizador de reações reversíveis ou irreversíveis de degradação e/ou formação de compostos químicos.

\section{CONCLUSÃO}

Por meio das análises termoanalíticas, cromatográfica e espectroscópica conclui-se que o armazenamento hermeticamente do óleo essencial de tomilho não provocou flutuações significativas do comportamento térmico e composição química do óleo. Os tratamentos do óleo essencial de tomilho em acondicionado aberto apresentaram estabilidade térmica otimizada nas amostras tratadas até $60^{\circ} \mathrm{C}$. A variação da entalpia foi amplificada com o aumento da temperatura de tratamento térmico possivelmente devido ao aumento do calor latente de cada amostra durante a transição de fase. Resultados obtidos por meio da CG e FTIR inferiram que a diminuição dos compostos majoritários e bandas obtidas nos grupos funcionais foi uma consequência direta do fenômeno de vaporização e degradação termogravimétrica do óleo essencial de tomilho.

\section{Agradecimentos}

Os autores agradecem à Financiadora de Estudos e Projetos (FINEP) pela aquisição e manutenção do equipamento de análise térmica e de cromatografia gasosa/espectrometria de massas.

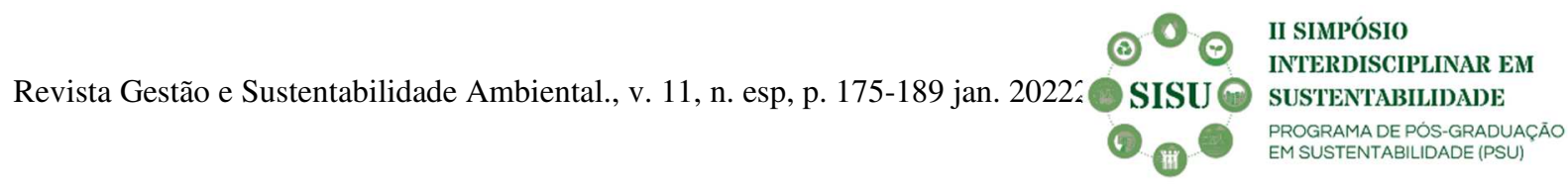




\section{Contribuições dos autores}
a) Conceituação: Angela Maria Picolloto e Cristiane Mengue Feniman Moritz.
b) Análise formal: Cristiane Mengue Feniman Moritz.
c) Metodologia: Angela Maria Picolloto, Angela Maria Ariati, Lidaiane Mariah Silva dos Santos Franciscato, João Marcos Azevedo Gomes dos Santos, Paulo Rodrigo Stival Bittencourt e Elina Basto Caramão.
d) Supervisão: Cristiane Mengue Feniman Moritz.
e) Validação de resultados: Angela Maria Picolloto e Paulo Rodrigo Stival Bittencourt.
f) Redação da minuta: Angela Maria Picolloto.
g) Redação, revisão e edição: Cristiane Mengue Feniman Moritz.

\section{Declaração de conflito de interesses}

Os autores declaram que não há conflitos de interesse existentes entre os autores e instituições parceiras neste estudo.

\section{REFERÊNCIAS}

ADAMS, R. P. Identification of essential oil components by gas chromatography/mass spectrometry, 4.1, 2017.

CANEVAROLO, S. V. J. Técnicas de Caracterização de Polímeros. Editora Artlibler. São Carlos, 2017. 69 p.

DEL RÉ, P. V.; JORGE, N. Antioxidant potential of oregano (Oreganum vulgare L.), basil (Ocimum basilicum L.) and thyme (Thymus vulgaris L.): application of oleoresins in vegetable oil. Food Science and Technology, v. 31, n. 4, p. 955-959, 2011.

DOLEA, D.; RIZO, A.; FUENTES, A.; BARAT, J. M.; FERNÁNDEZ-SEGOVIA, I. Effect of thyme and oregano essential oils on the shelf life of salmon and seaweed burgers. Food Science and Technology International, v. 24, n. 5, p. 394-403, 2018.

HUANG, L.; WANG, Y.; LI, R.; WANG, Q.; DONG, J.; WANG, J.; LU, S. (2021). Thyme essential oil and sausage diameter effects on biogenic amine formation and microbiological load in smoked horse meat sausage. Food Bioscience, v. 40, 2021.

IANOSHIRO, M. GIOLITO, I. Fundamentos da termogravimetria, análise térmica diferencial e calorimetria exploratória diferencial. Giz Editorial, 2004, 82p.

MAIER, S. A. Plasmonics: fundamentals and applications. Springer, 2004.

NISTBOOK. National Institute of Standards and Technology (NIST). Nist Livro de Química na web, SRD 69. 2020. https://webbook.nist.gov/chemistry/.

PICOLlOTO, A. M.; ARIATI, M.; FRANCISCATO, L. M. S. S.; BITTENCOURT, E. B. C.; SAKAI, O. A.; MORITZ, C. M. F. Chemical and thermoanalytical characterization of the pink pepper

Revista Gestão e Sustentabilidade Ambiental., v. 11, n. esp, p. 175-189 jan. 2022^




\section{GESTÃO \& SUSTENTABILIDADE AMBIENTAL \\ ¿unisul}

(Schinus terebinthifolius Raddi) seeds essential oil. Revista Mundi Engenharia, Tecnologia e Gestão, v. 5, n. 7, p. 286-301, 2020.

RIBEIRO-SANTOS, R.; ANDRADE, M.; SANCHES-SILVA, A.; DE MELO, N. R. Essential oils for food application: natural substances with established biological activities. Food and bioprocess technology, v. 11, n. 1, p. 43-71, 2018.

SHARMA, S.; BARKAUSKAITE, S.; JAISWAL, A. K.; JAISWAL, S. Essential oils as additives in active food packaging. Food Chemistry, v. 343, 2020.

SHEN, Z.; KAMDEM, D. P. Development and characterization of biodegradable chitosan films containig two essentials oils. International Journal of Biological Macromolecules, v. 74, p. 289-296, 2015.

SIQUEIRA, M, S. C., BRITO, D. R., SILVA, F. O. C. A utilização do óleo essencial de tomilho (THYMUS VULGARIS) como recurso fitoterápico para acne. In Revista Científica da FHO, Uniararas, v. 3 (1). 2015, 63-72. 